\title{
PROCESOS DE INTEGRACIÓN DE PERSONAS SOLICITANTES DE PROTECCIÓN INTERNACIONAL Y REFUGIADAS EN LA CIUDAD DE ALICANTE. CRUZ ROJA ESPANOLA ${ }^{1}$ \\ INTEGRATION PROCESSES OF PERSONS SEEKING INTERNATIONAL PROTECTION AND REFUGEES IN THE CITY OF ALICANTE. SPANISH RED CROSS
}

\author{
Natalia Araujo Rocca \\ Cruz Roja Española en Alicante \\ nar.alicante@cruzroja.es \\ Recibido: 11/5/2010 \\ Aceptado: 3/6/2010
}

\begin{abstract}
Resumen:
La llegada a un país de residencia diferente al de origen implica, para cualquier persona, un reajuste y adaptación a la nueva realidad, y también a la propia sociedad receptora, no exenta de dificultades.

Este artículo expone en primer lugar, las conclusiones acerca de cuáles son y como intervienen determinados condicionantes (internos y externos), influyentes en el proceso de integración social de las personas solicitantes de protección internacional en España, en un entorno urbano. En segundo lugar, se aborda la realidad encontrada por este colectivo durante dicho proceso de integración social, en el contexto concreto de la ciudad de Alicante.

Palabras clave: procesos; integración; condicionantes; áreas; protección; social.

\footnotetext{
${ }^{1}$ Resumen sobre la intervención desarrollada en la mesa de trabajo: "El refugio y asilo en España", incluida en el programa del "I Seminario de Migraciones Forzadas. Población desplazada y refugiada en entornos urbanos", celebrada en la Universidad de Alicante los días 15,16 y 17 de marzo de 2010.
} 


\begin{abstract}
:
The arrival at a country of residence different from that of origin implies for any person, a readjustment and adaptation to the new reality, and even for society itself which receive, does not exempt of difficulties.

Firstly, this article expounds the conclusions about how certain conditions intervene and witch are these (internal and external), influential in the course of social integration of the persons seeking international protection in an urban environment in Spain. Secondly, it deals with the reality found by this group during the above mentioned process of social integration, in the specific context of Alicante.
\end{abstract}

Keywords: processes; integration; determining; areas; protection; social.

\title{
INTRODUCCIÓN
}

Cruz Roja Española en Alicante viene desarrollando desde hace años un Programa de Refugiados, con el que se pretende, desde una perspectiva social y humanitaria, dar respuesta a las situaciones de necesidad que las personas solicitantes de asilo y refugiadas presentan en nuestra provincia. Desde este marco de actuación, se desarrollará una breve aproximación al proceso de integración de una persona solicitante de asilo, llegada a la provincia de Alicante, siempre basada en las experiencias y aprendizajes adquiridos a través del trabajo diario realizado en el Departamento de Inmigrantes y Refugiados. Es decir, se trata de unas conclusiones de carácter empírico, entendiendo que las realidades variarán según el contexto de residencia.

En primer lugar se expondrá un breve repaso a los condicionantes que entendemos están influyendo en el proceso de integración social de una persona solicitante de protección internacional en un entorno urbano. Se destacan únicamente los aspectos que entendemos de mayor relevancia y que influyen de una manera más decisiva en dicho proceso. Estos aspectos se presentan diferenciados en factores internos y externos a la persona, que pueden representar tanto fortalezas como debilidades en la adaptación a un nuevo país.

En segundo lugar se expondrán las diferentes situaciones y realidades que encuentra una persona solicitante de protección internacional en la ciudad de Alicante, refiriéndonos principalmente a personas que son o han sido participantes en nuestra institución, como usuarios del Programa de Refugiados.

\section{CONDICIONANTES DE LOS PROCESOS DE INTEGRACIÓN SOCIAL}

Como factores internos destacan, en primer lugar, la nacionalidad unida al idioma, factores claves para comprender los diferentes puntos de partida de una persona recién llegada a un país. Se debe tener en cuenta que el desco- 
nocimiento del idioma utilizado en una sociedad limita las posibilidades de comunicación con sus habitantes. La cultura, costumbres, tradiciones, etc., varían de unos países a otros, pero cuanto mas similares sean al del país de acogida, más fácil puede resultar la integración en su sociedad, que sitúa al "Otro" como alguien menos lejano.

El status familiar del migrante supone también un punto de partida diferente. Por ejemplo, el proceso de inclusión social de una mujer con cargas familiares será muy diferente al de un hombre joven, soltero y sin descendientes. Se comprende que el diferente rol asociado a un status implica un determinado posicionamiento social, responsabilidades, limitaciones, apoyos emocionales, vínculos sociales, etc.

El nivel educativo y formativo previo, e incluso el status social de la persona en el país de origen, puede suponer un factor de facilitación o, por contrario, obstaculizar el proceso de integración social, al implicar por ejemplo el conocimiento/desconocimiento del país acogida o de su legislación y políticas en materia de Asilo y derechos sociales, facilidad o no en la búsqueda y utilización de recursos, habilidades como conocimiento de idiomas, de utilización de tecnologías. Por otra parte, la cualificación profesional y experiencia laboral previa condicionan el acceso al mercado laboral.

Las expectativas previas de la persona llegada a un nuevo país relativas al grado de protección que obtendrá o la acogida que va a recibir, la posibilidad de autoabastecer sus necesidades, etc, pueden suponer también una gran diferencia a la hora de asimilar los cambios y adaptarse al nuevo entorno. Las altas expectativas resultan más difíciles de satisfacer, pudiendo generar sentimientos negativos que dificulten aún mas la adaptación a una realidad concreta.

En este mismo sentido, el trayecto migratorio, (que puede haber sido de corta o larga duración, con experiencias en el camino con una mayor o menor carga traumáticas), sumados a los motivos de salida de su país de origen, podrá implicar un mayor sufrimiento y repercusiones negativas a nivel físico y psicológico. Esto incide directamente en las capacidades y habilidades que una persona debe movilizar interiormente para enfrentarse a un nuevo reto, como es el adaptarse a un nuevo país, cultura, costumbres e incluso idioma.

Como ya he mencionado anteriormente, el motivo del abandono de su país y por tanto, de solicitar protección internacional, es uno de los factores internos más decisivos en relación con el proceso de integración del solicitante de asilo. Encontramos historias más o menos traumáticas, con diferentes niveles de riesgo vivido y sentido, como ser y/o sentirse victimas de maltrato, torturas, amenazas o cualquier otro tipo de violencia. Todas estas experiencias condicionan en gran medida el bienestar psíquico y físico de la persona. 
Relacionado con esto último se encuentra el estado de salud, tanto física, como emocional y psicológica. Ya se ha abordado la importancia que en los procesos de integración social tienen los sentimientos y estados generados en una persona tras la huida de su país: frustración al no conseguir cumplir las expectativas, estrés migratorio, los diferentes estilos de respuesta con las que la personas afronta las adversidades, la interpretación que hace de la realidad vigente, el estrés cultural, etc. Tampoco se debe olvidar que una situación de cambio como resulta la huida de un país y, al mismo tiempo, la adaptación a una nueva sociedad, puede suponer cambios a nivel intrafamiliar/cambio de roles, que pueden desestabilizar la dinámica funcional de la familia.

Las enfermedades físicas e incapacidades surgidas tras la migración o previas, pueden implicar mayores dificultades para la inserción social, mayores cuanto más limitantes resulten.

En cuanto a los factores externos, en primer lugar podemos hablar del status jurídico de la persona solicitante de protección internacional. Los diferentes tipos de documentación que el Gobierno Español otorga a dichas personas (por ejemplo, al incluir o no un permiso de trabajo), influyen de forma decisiva en diferentes aspectos de la vida de una persona, facilitando o dificultando el logro del autoabastecimiento de necesidades básicas, o el acceso a diferentes recursos sociales (como puede ser al Servicio Nacional de Empleo y su oferta formativa).

No se debe olvidar un factor absolutamente determinante en el proceso de integración de cualquier sujeto llegado a un nuevo país: se trata de las características de la sociedad de acogida, con su particular cultura, su sistema de creencias, estereotipos, políticas, leyes, sistemas de protección etc. El rechazo social que suelen generar las diferencias aumenta cuanto mayores sean éstas, algo que también se ve condicionado por el nivel económico, social y cultural de la persona que llega a nuestro país. La información acerca del estatuto de refugiado o solicitante de protección internacional que tenga la población donde se resida puede implicar falta de sensibilidad acerca de la idiosincrasia del colectivo y sus necesidades especiales de protección.

El apoyo social que pueda recibir una persona perteneciente a este colectivo, resulta un factor facilitador para lograr una integración social mas completa. Esto implica tanto a las propias redes sociales más cercanas (familia, amigos, compatriotas, otros residentes del Centro, ex-residentes) como al resto de la sociedad de acogida (Cruz Roja, ONG'S, Servicios Sociales municipales y comunitarios...).

La situación económica y del mercado de trabajo de la sociedad de acogida también supone un condicionante a la hora de lograr la inserción social de 
cualquier persona, al ser el desempeño de un trabajo la forma de lograr ingresos económicos y por tanto, la necesaria autonomía. Durante el año pasado, ha habido un aumento de las tasas de desempleo, algo que ha repercutido en los colectivos más vulnerables de la sociedad española, en el que están incluidos las personas solicitantes de protección internacional y refugiadas.

También en relación a la sociedad de acogida, condiciona el proceso de integración de una persona la estructura de sus instituciones. Con esto nos referimos al sistema de sanidad, educación, empleo, vivienda, Servicios Sociales, etc. Cada uno de ellos posee sus propios requisitos de acceso a sus servicios. En España la normativa en materia de protección internacional y las directivas europeas han marcado los derechos que una persona refugiada o solicitante de asilo tiene en cuanto al acceso a los recursos sociales públicos.

Por último, mencionar que la participación social supone un factor integrador. La inclusión en asociaciones, entidades sociales o la práctica de voluntariado, favorecen el conocimiento del país, la formación de redes sociales, el reconocimiento de derechos, la visibilidad del colectivo, la eliminación de estereotipos o la utilización de recursos normalizados.

\section{ÁREAS IMPLICADAS EN LOS PROCESOS DE INTEGRACIÓN SOCIAL}

En este punto se abordarán algunas realidades encontradas en la ciudad de Alicante por personas solicitantes de protección internacional (asilo y protección subsidiaria) y personas que ya poseen el estatuto de refugiado. Dichas realidades serán abordadas desde diferentes áreas, entendidas como las más significativas.

Con respecto al área jurídica, la atención en la provincia de Alicante a las solicitudes de protección internacional es prestada desde ONG s (por ejemplo en Cruz Roja existe un servicio especializado en materia de asilo). La Brigada Provincial de Extranjería y Fronteras, en la ciudad de Alicante, recibe y tramita las solicitudes que se presentan en la provincia.

El área de atención de carácter psicológico resulta muy relevante en el trabajo con las personas solicitantes de protección internacional y refugiadas. Muchas de ellas encuentran dificultades para afrontar las vivencias pasadas y presentes, el estrés migratorio, las pérdidas (reales y simbólicas) de referentes, de familiares o de forma de vida. A esto puede unirse el desconocimiento de códigos culturales o del idioma. Por todo ello, una gran mayoría de personas puede necesitar apoyo profesional para ir asimilando los cambios o, en los casos mas graves, ir superando los diferentes trastornos que puedan surgir. La respuesta a esta necesidad se encuentra cubierta en Alicante por la oferta de ONG s, y desde las USM, pero generalmente no es específica para 
este colectivo. Cruz Roja Española en Alicante, dentro de las prestaciones que incluye el Programa de Refugiados que desarrolla, presta atención psicológica especializada.

En cuanto al área de atención social, encontramos que en Alicante la cobertura de la necesidad de información, orientación y asesoramiento a personas solicitantes de asilo/refugiadas, está cubierta a través de diferentes recursos tanto públicos como privados: Centros Sociales, Oficinas AMICS y ONG s especializadas en la atención a personas inmigrantes. Todos ellos cuentan con servicios donde un profesional atiende dichas demandas.

La cobertura de necesidades básicas (de manutención, alojamiento y otras) se encuentra satisfecha si la persona es residente en el Centro de Migraciones que Cruz Roja Española mantiene en la ciudad. Sin embargo, si viven fuera de él y requieren apoyo para cubrir estas necesidades, encuentran ciertas dificultades para recibirlo de un modo tan amplio. El acceso a los recursos públicos que en la ciudad de Alicante prestan este tipo de atención se ve limitado en ocasiones por el desconocimiento de la existencia y de los servicios de los mismos, o dificultado por la masificación existente en algunos de ellos (que atienden las zonas de la ciudad con población más desfavorecida, y donde suele residir este colectivo).

Cruz Roja Española cuenta con un Convenio con la Administración pública (Ministerio Trabajo e Inmigración) que regula la atención que se presta tanto en el Centro de Migraciones que Cruz Roja tiene en Alicante como en el proyecto que también desarrolla en la ciudad: Centros y Oficinas de Atención Integral al Refugiado. En ambos se presta entre otras, la atención social necesaria a las personas solicitantes de protección internacional y refugiadas residentes o no en el Centro de Migraciones.

Respecto al área de vivienda, el colectivo de inmigrantes es uno de los que encuentra mayores dificultades de acceso. Es frecuente encontrar que, debido a la difícil situación económica de muchos de ellos, sus posibilidades de alojamiento digno son muy escasas, debiendo instalarse en condiciones frecuentes de hacinamiento en viviendas deterioradas. Además, existe en la ciudad de Alicante, segregación residencial en unos pocos barrios, de las personas inmigrantes y minorías étnicas.

El solicitante de asilo/refugiado padece dificultades al salir de Centro de Migraciones (de igual forma si nunca ha residido en él) en cuanto al acceso a la vivienda, debido principalmente a la carencia de recursos económicos autónomos y estables. Ante ello se encuentra en la necesidad de alquilar una habitación en una vivienda compartida, puede que en condiciones precarias o de hacinamiento, víctima además con frecuencia de abusos como el no con- 
tar con un contrato de alquiler y por tanto quedar totalmente desprotegido de derechos. En muchas ocasiones en que se arrienda una habitación, no se permite empadronarse en ella, algo que es requerido para el acceso a numerosos recursos sociales/sanitarios de carácter público.

Si hablamos de familias es aún más complicado, pues apenas hay oferta de alquiler de habitaciones compartidas donde permitan la presencia de menores.

Algunas personas solicitantes de asilo/refugiadas poseen una red de apoyo a través de la cual pueden encontrar una vivienda más cómoda, compartida con menos personas, con conocidos, o solas, etc., pero nuestra experiencia indica que ésta no es generalmente la situación mas frecuente.

El apoyo en la búsqueda de vivienda es prestado desde diferentes ONG s, Cruz Roja tiene un servicio específico, que al igual que el de otras entidades de la ciudad, trabaja por facilitar el acceso a una vivienda en condiciones dignas.

Si se aborda el área de empleo en la ciudad de Alicante, encontramos la existencia de diversos recursos sociales (públicos y privados) de apoyo. Numerosas ONG s trabajan esa área además del Servicio Estatal Valenciano de Fomento del Empleo. Cruz Roja Española en Alicante posee un Servicio de Empleo, en el que se inserta un proyecto específico para personas solicitantes de protección internacional y refugiadas.

Cuando una persona nacional de otro país llega a la ciudad, generalmente no tiene conocimiento previo sobre la existencia de los recursos públicos y privados de acceso al empleo. Tampoco del mercado laboral español e incluso de los códigos culturales necesarios para acceder a un empleo. La mayoría de estas situaciones cambian rápidamente según va avanzando el tiempo de residencia en la ciudad de Alicante, y la persona va ampliando su red social de apoyo, que le guía en el manejo de recursos. También esta situación varía gracias a las actuaciones de apoyo que realizan las entidades sociales en este ámbito.

Las personas solicitantes de protección internacional a quienes se les ha facilitado el permiso de trabajo (éste se consigue tras haber pasado seis meses desde la admisión a trámite de la solicitud y mientras no haya habido resolución de la misma), y las personas refugiadas (cuyo estatuto permite trabajar), encuentran en estos momentos enormes dificultades para acceder a cualquier contrato de trabajo. Esta situación implica la dependencia del apoyo social para cubrir sus necesidades básicas de subsistencia. En muchas ocasiones si se accede a un empleo, éste puede ser en condiciones de precariedad (sin contrato legalizado, remunerados de forma escasa, de carácter eventual, jornadas excesivas...).

El derecho a ser contratado y desempeñar un empleo remunerado de forma ajustada a la legalidad se extingue si la persona ve rechazada su solicitud de asilo, por lo que si se encuentra trabajando con contrato, sus emplea- 
dores se ven obligados a rescindírselo. También se debe mencionar que debido a la falta de oferta o del reconocimiento de sus titulaciones profesionales en España, este colectivo encuentra que deben acceder a empleos muy diferentes a los que realizaban en sus países de origen ( peor valorados y retribuidos).

Es necesaria la capacitación profesional, debido a la falta de formación de muchas personas pertenecientes al colectivo. La ciudad de Alicante tiene oferta formativa en este sentido, pero insuficiente en la práctica, debido a la gran demanda social. Conviven los cursos de las ONG s con la propuesta pública a través del Servicio Estatal Valenciano de Fomento del Empleo.

Por último, refiriéndose a éste área, mencionar la importancia que para las personas solicitantes de protección internacional y refugiadas (al igual que para el resto de la población de la ciudad) tiene el funcionamiento de las redes informales de apoyo en la búsqueda de empleo.

Si nos referimos al área de educación y cultura, no se puede olvidar que los condicionantes educativos y culturales son importantes para la integración de las personas solicitantes de protección internacional y refugiadas. Ya se ha mencionado su relación con el acceso al empleo y a los recursos sociales de la ciudad. Existen dificultades de adaptación a un entorno cultural desconocido, que son abordadas de diferentes formas y a través de recursos distintos (por parte de recursos públicos y privados en la ciudad). Por ejemplo desde Cruz Roja Española se ofrece formación en este sentido a través de una amplia oferta de talleres de conocimiento del entorno y costumbres, legislación, normas cívicas, sistemas y recursos de empleo, sociales, sanitarios o educativos, clases de castellano, de valenciano, etc., para el traslado de la información básica y necesaria que pueda facilitar la integración social del colectivo en la ciudad. Debe hacerse una mención especial a la facilidad para acceder a clases de español en la ciudad, al existir una amplia oferta en múltiples asociaciones y servicios públicos, de carácter gratuito o muy económico.

Además, ya que la integración social es un proceso en el que intervienen dos factores (la persona que llega y la sociedad de acogida) no se debe olvidar la importancia de las acciones de sensibilización hacia la ciudadanía valenciana sobre la inmigración como fenómeno positivo y, para el conocimiento de la condición de solicitante de asilo y refugiado. En la provincia de Alicante, Cruz Roja desarrolla este objetivo desde talleres de sensibilización en centros educativos, participación en Ferias como INTERPLURAL, celebración de actos para conmemorar el Día del Refugiado, organización o participación en Jornadas Interculturales, en charlas y eventos informativos, etc. Todas estas actividades promueven los contactos interculturales para facilitar conocimiento mutuo. También desde otras ONG s y los servicios públicos se 
realizan diversas actividades que dan lugar al contacto entre colectivos (colectivo inmigrante, no tiene porque ser el de solicitantes de protección internacional/refugiadas), intercambio de información, vivencias de experiencias comunes a través de jornadas lúdicas, torneos deportivos, jornadas gastronómicas. etc.

En general, no ha habido problemas para que los hijos e hijas de las personas solicitantes de asilo tengan acceso a la educación, siempre y cuando estén en edad de escolarización obligatoria. Además, dicha incorporación cuenta con apoyo personalizado para los menores dentro del centro escolar a través de acciones de refuerzo individualizado. Cruz Roja Española en Alicante cuenta también con clases de apoyo escolar a menores extranjeros, al que acceden los menores hijos de los solicitantes de protección internacional y refugiadas si se valora necesario. En el caso de los menores en edad de escolarización no obligatoria, su incorporación depende del número de plazas disponibles de los centros educativos. Por tanto, en la localidad de Alicante existen problemas relacionados con la necesidad de matriculación de hermanos en centros educativos diferentes o la imposibilidad de matriculación debido a la carencia de plazas.

En particular, para escolarizar a los menores de 3 años, la realidad se encuentra plagada de obstáculos: la oferta pública en Alicante no cubre la demanda existente; además, debe pagarse un importe mensual muchas veces no asequible para las personas interesadas, o los centros (en número muy escaso) se encuentran a veces muy lejos del domicilio del menor por lo que el gasto de transporte o tiempo empleado para llegar al centro dificulta la búsqueda de empleo o limita la incorporación. La oferta de centros privados es muy amplia, pero el coste económico es mayor. La incorporación a estudios universitarios, formación profesional, etc., no suele darse, ya que durante los primeros años de estancia en la ciudad las personas solicitantes de protección internacional y refugiadas centran sus esfuerzos en la forma de conseguir recursos económicos o en hacer frente a dificultades emocionales.

En cuanto al área de Sanidad, cuando una persona llega para residir de forma estable en la ciudad de Alicante procedente de otro país, una de las primeras gestiones que debe realizar es la tramitación de su tarjeta sanitaria, que le permite el acceso al sistema nacional de salud y sus servicios. En ocasiones no es posible obtener la tarjeta sanitaria de forma inmediata, aunque esta situación se resuelve siempre en un plazo breve de tiempo. Mientras no está incluida como beneficiaria en la Seguridad Social, una persona solicitante de protección internaciones puede ser atendida desde las ONG s. Si se cuenta con el estatuto de refugiado siempre se recibe la protección sanitaria del sis- 
tema público español. Debe mencionarse la gran facilidad para tramitar la tarjeta sanitaria en algunos casos: mujeres embarazadas, menores o personas que padecen alguna enfermedad que hace necesario que reciban tratamiento y control de forma inmediata. Una vez accedido al sistema de salud, las dificultades que pueden encontrarse son: para comunicarse (debido a la barrera lingüística) o el desconocimiento de forma de entender la salud (diferentes en cada país) y la estructura del sistema en Alicante. Este sistema y la protección que presta son muy bien valorados generalmente por las personas solicitantes de protección internacional y refugiadas.

En cuanto a las enfermedades que este colectivo manifiesta, muchas veces se encuentran asociadas a sus vivencias pasadas (el estrés migratorio, la historia de padecimientos en su país de origen o en el tránsito hasta llegar a Alicante) o por la situación actual (incertidumbre acerca de su futuro, lejanía de referentes familiares o culturales, etc.). Se trata por ejemplo de trastornos de estrés postraumático, síndrome de Ulises, trastornos de ansiedad, de sueño. Por otro lado, con frecuencia se detectan en la ciudad enfermedades que ya tenían estas personas en sus países de origen (conocidas o no por ellos). En ocasiones aparecen reticencias a reconocer estas enfermedades como propias.

Desde el Programa de Refugiados que desarrolla Cruz Roja Española en Alicante se llevan a cabo numerosas acciones para el cuidado de la salud, a través no sólo del apoyo para la tramitación de tarjetas sanitarias, sino también de la realización de charlas y talleres grupales o la atención individualizada a los residentes de las viviendas y las personas que viven fuera de ellas para la promoción de la salud: apoyo en el cuidado del menor, información sobre sistema de salud público, nutrición, hábitos de higiene, prevención de enfermedades... prestaciones farmacéuticas, pago de servicios de óptica, odontólogo, ortopedia...

En referencia al área de mujer, las mujeres inmigrantes y solicitantes de protección internacional y refugiadas pueden contar con diversos servicios dirigidos de forma específica (o dirigidas a la población general) a atender sus necesidades. Éstos se facilitarán tanto a través de diferentes recursos públicos como privados, y abarcan los diferentes aspectos: salud sexual y reproductiva, atención psicológica, jurídica, laboral, oferta formativa, etc. Desde el Programa de Refugiados y el Servicio de Empleo de Cruz Roja Española en Alicante se trabaja la prevención de situaciones de riesgo y el cambio de algunas realidades en que se encuentran las mujeres solicitantes de protección internacional y refugiadas. Se trabaja especialmente para promover la autoestima y la autosuficiencia de la mujer, a través de atenciones tanto de carácter psicológico como educativo, en intervenciones grupales e individuales. 
Por último se aborda el área de Participación y Asociacionismo. La participación social de cualquier colectivo permite su visibilidad, la disminución de muchos estereotipos, la facilitación de formación de redes de apoyo y previene situaciones de soledad, depresión y exclusión social. Como antes he mencionado, existen en la ciudad de Alicante diversas oportunidades de participación social para las personas solicitantes de protección internacional y refugiadas: en ONG s, en asociaciones de inmigrantes, asociaciones vecinales, comisiones de Hogueras y Barracas...

Mención aparte merece la participación a través del voluntariado social. En Alicante, Cruz Roja cuenta con numerosas personas inmigrantes y algunas solicitantes de protección internacional y refugiadas (muchos de ellos han sido anteriormente usuarios de los servicios) realizando labor voluntaria en la ciudad. Todos ellos tienen en común un interés por retribuir de alguna forma el apoyo que en algún momento recibieron, el sentirse parte importante y necesaria de una comunidad.

\section{CONCLUSIÓN}

Como se ha analizado en esta exposición, son numerosos los factores que inciden en el proceso de integración social de una persona solicitante de protección internacional o refugiada, y más aún las dificultades que encuentran hasta poder alcanzar una vida satisfactoria y digna en nuestra ciudad. Pero no se debe olvidar que también son muchos los equipos profesionales y personas voluntarias, las entidades, asociaciones y recursos que en Alicante trabajan para que la acogida y la protección que éste colectivo recibe sean adecuadas y suficientes.

Cruz Roja Española en Alicante trabaja con ese fin, y bajo sus principios fundamentales de actuación, prestando atención a las personas solicitantes de protección internacional y refugiadas que llegan a la ciudad. 